\title{
ATLAS Measurements Sensitive to the Proton Structure
}

\author{
P. J. Bussey ${ }^{1, a}$ \\ on behalf of the ATLAS Collaboration \\ ${ }^{1}$ SUPA, School of Physics and Astronomy, University of Glasgow, Glasgow G12 8QQ, United Kingdom
}

\begin{abstract}
This talk presents a survey of ATLAS measurements at the LHC that are sensitive in various ways to the parton distribution functions of the proton.
\end{abstract}

\section{Introduction}

The parton structure of the proton plays a central role in the study of all particle interactions at the LHC that take place with large momentum transfer. An accurate understanding of such processes therefore requires reliable determination of the parton distribution functions (PDFs) of the proton. These are essentially derived from experiment. In the present talk we survey a number of measurements made with ATLAS [1] that are sensitive to the proton PDFs, which are presented in terms of models that have been constructed in various ways, based on measurements at the LHC but also at HERA, Fermilab and other laboratories. Different processes depend on the proton structure in different ways. The study of inclusive jets, dijets and trijets in high energy events provides insight into the gluon PDF of the proton. Drell-Yan processes are sensitive to sea quark distributions, while processes in which $Z$ and $W$ bosons are emitted can also give information on sea quark distributions.

\section{Inclusive jets, dijets and trijet states.}

In a recent study of high-momentum inclusive jet production [2], ATLAS first verify that the NLOJET ++ model is capable of giving a good general description of the jet production over all accessible rapidity and transverse momentum $\left(p_{T}\right)$ values, using the standard CT10 PDF set. This enables different PDF models to be compared with confidence and gives an extensive coverage of the standard kinematic quantities $x$ and $Q^{2}$. In fig. 1, the ratio of the measured and theoretical cross sections is shown for a variety of published PDF sets. Of these, CT10, MSTW 2008, NNPDF 2.1 and HERAPDF 1.5 are found to do well over nearly all the region, but ABM11 (with five flavours) shows significant discrepancies at central rapidity values, where the fit probabilities are low. Specific references to the relevant PDF sets can be found in the cited ATLAS publications.

Dijet [3] and trijet [4] final states have also been studied, and again the general form of the cross section is first shown to be well modelled by the NLOJET Monte Carlo. Figure 2 shows a selection of the comparison of the results with proton structure models, in bands of the mean rapidity difference $y^{*}$ of the dijets, and an analogous rapidity measure $Y^{*}$ of the trijets. The same models that succeeded well

\footnotetext{
ae-mail: peter.bussey@glasgow.ac.uk
} 



Figure 1. Transverse momentum of inclusive jets at ATLAS [2], compared with various models of the proton structure. Theory uncertainties are shown by coloured bands.

for the inclusive jets succeed well also for these cases, with the addition of epATLjet 13, which was based on a dedicated fit to HERA and ATLAS data. ABM 11 is unsatisfactory, as before. Electroweak corrections were applied to the dijet measurements but were unavailable for the trijets.

In principle, inclusive prompt photon cross sections should also show sensitivity to the proton structure. In practice, this is quite low and although the measured cross sections are all described well by the MCFM model, there is little differentiation between different PDF sets at present.

\section{Top quark production}

The production of pairs of top quarks provides a different perspective on the proton structure, and high statistics are available at the LHC. There are many parameters that can be measured, and several 

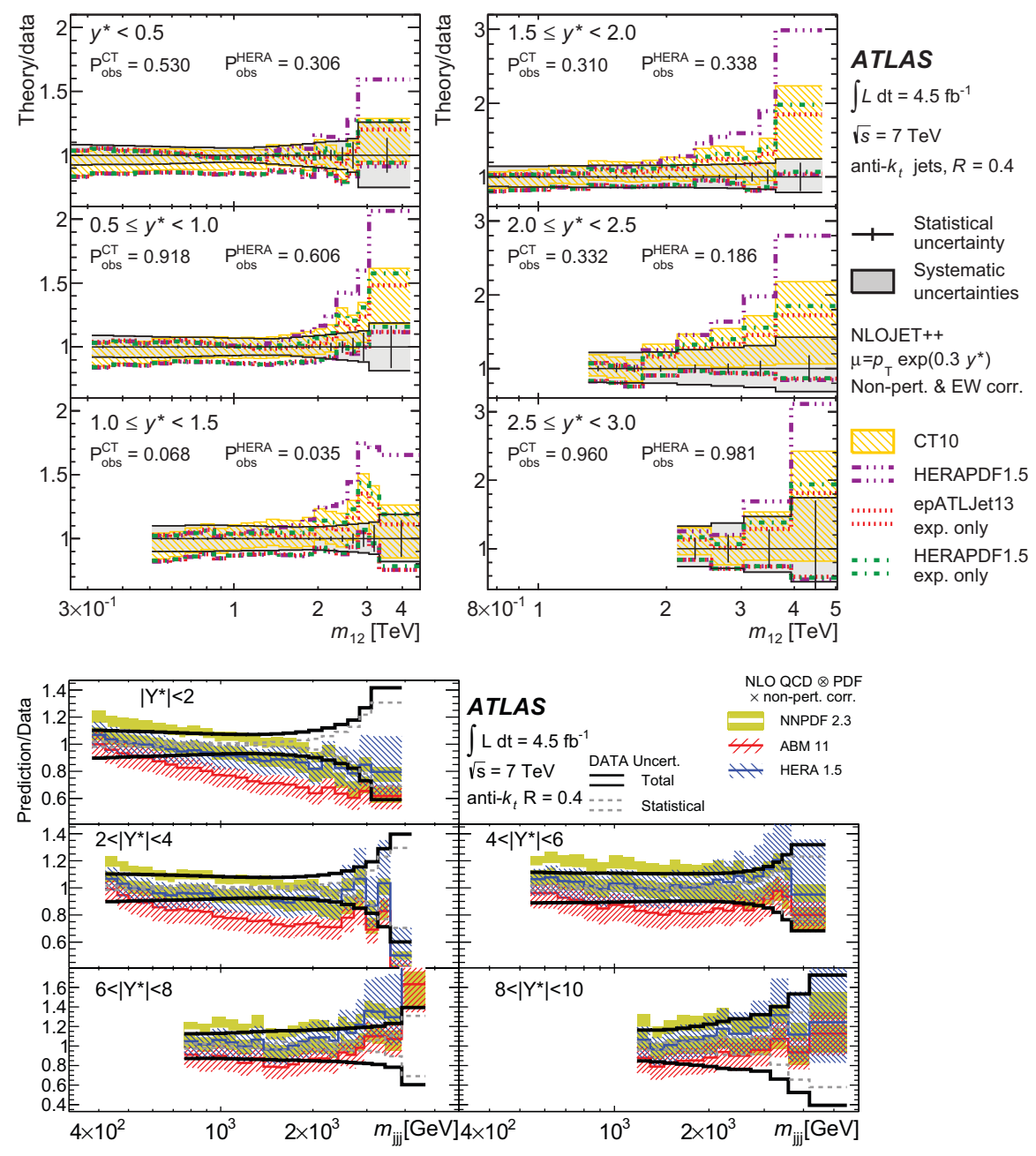

Figure 2. Mass of dijets [3] (upper) and trijets [4] (lower) at ATLAS, compared with various models of the proton structure. Theory uncertainties are shown by coloured bands.

models of the dynamics of the process. Of these, ALPGEN, MC@NLO, POWHEG (all using HERWIG) and POWHEG (using PYTHIA) are all found to be satisfactory, although the agreement with data is not perfect [5]. An example is the mass of the top-antitop system, which can be reconstructed in various decay modes of the $W$ bosons in the decays. ATLAS have studied this process using final states defined with a lepton and four jets, with at least one $b$-meson tag. Figure 3 (left) shows that three standard models evaluated using NLO theory (CT10 NLO, MSTW 2008 and NNPDF 2.3) tend to lie above the data at high values of the mass, while HERAPDF 1.5 gives a good description over the whole range. Other variables do not discriminate well between the models.

These studies have been continued using 8-TeV LHC data. A typical example is shown in fig. 3 (right) where the rapidity of single top mesons is compared with theory, again at the NLO level. In 



Figure 3. Left: mass of $t \bar{t}$ system produced at ATLAS [5], compared with various models of the proton structure. Right: rapidity of top mesons.

this case, all the theories except HERAPDF 2.0 are satisfactory. When other variables are plotted, slightly different results are obtained; thus CT10nlo is found to be less satisfactory than the others when the rapidity of the top pair and of its boost are considered.

Other pairs of high-energy leptons are available for study. However the production of electronpositron pairs by the Drell-Yan process gives little differentiation between the available proton models at masses above $116 \mathrm{GeV}$, and all are found to give cross sections lying lower than the data [6]. At lower masses the data and theory are in agreement.

\section{$4 Z$ and $W$ production}

$Z$ bosons are produced copiously at the LHC, but the inclusive $Z$ cross section is poorly described by NLO theory as a function of the $p_{T}$ of the $Z$ [7]. In principle this process could be used to study the proton structure, but in practice the dynamics still need to be better modelled and understood.

The situation improves when further constraints are applied to the process under study, and the production of a $Z$ accompanied by $b$-jets is much more favourable [8]. Figure 4 shows (upper) that different models predict significantly different values for the cross section, but that MFCM and aMCNLO are satisfactory. At present, the scale uncertainties in the models prevent discrimination between different choices of PDF (lower figure), but there is potential for improvement using higher-order calculations. At present the MSTW 2008, NNPDF 2.3 and CT10 PDFs may all be regarded as satisfactory, when used with an appropriate model of the production dynamics.

The measurement of $W / Z$ ratios provides information that can be used to extract the elusive strange quark density in the proton. In specifying the density of the strange quark relative to the down quark as $r_{s}=0.5(s+\bar{s}) / d$, a suppression factor of 0.5 has been commonly employed. ATLAS have measured this ratio to be close to 1.0 [9], which is compared to several proton PDF models in fig. 5.

The production of $W$ mesons accompanied by a charm particle is capable of giving further information [10]. The charm quark is normally of the opposite sign to the $W$, and to remove backgrounds 

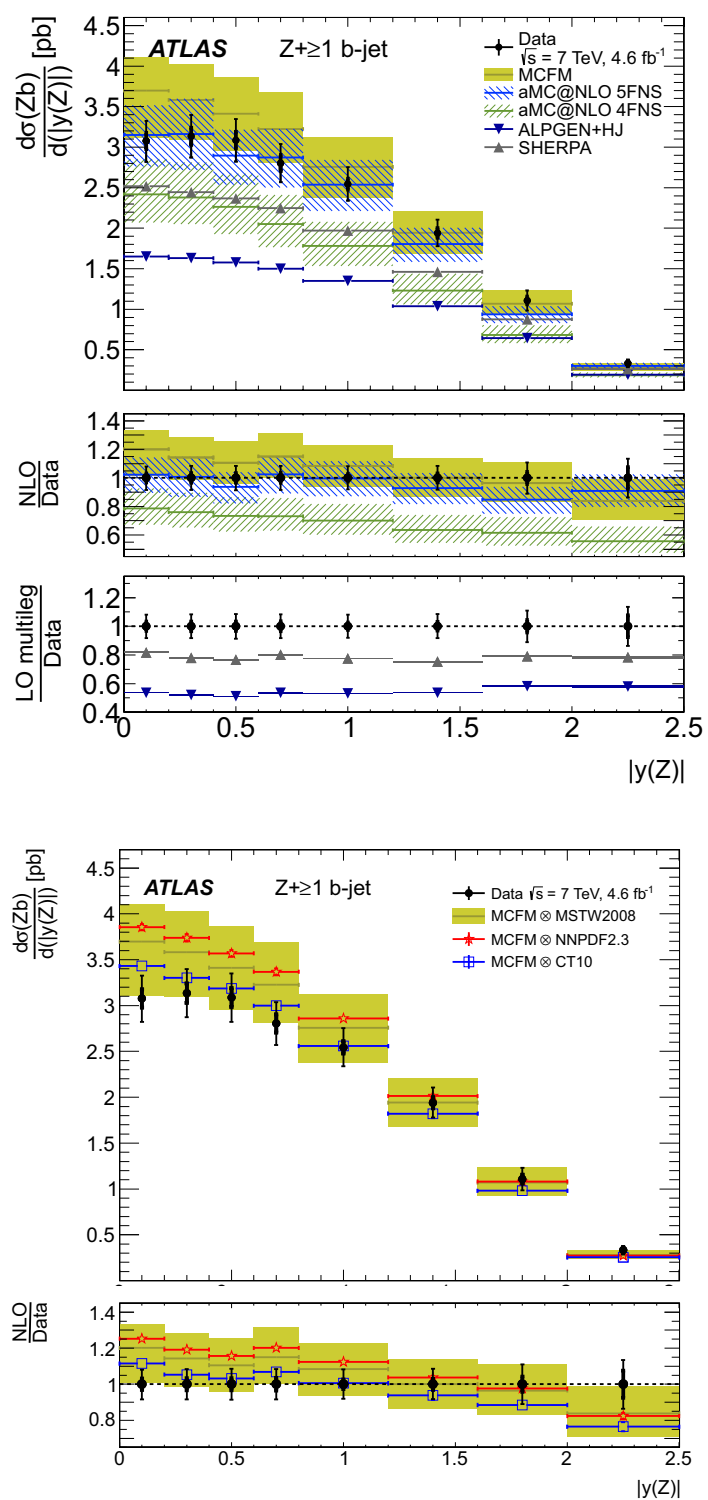

Figure 4. Rapidity of $Z$ accompanied by $b$-jets [8], compared with various models of the production process (upper) and proton structure (lower). Scale-dominated theoretical uncertainties are shown by coloured bands.

the same-sign backgrounds are subtracted. Results for two channels are shown in fig. 6, giving a certain amount of discrimination between different PDF models. There is scope for improvement by achieving smaller uncertainties both on the data and on the theory. The HERAFITTER program has been run using these data, and favours an unsuppressed strange quark contribution.

The simultaneous measurement of $W, Z$, and $t \bar{t}$ cross sections is capable of providing an important new set of viewpoints both on the production of these final states and of the proton structure [11]. As 


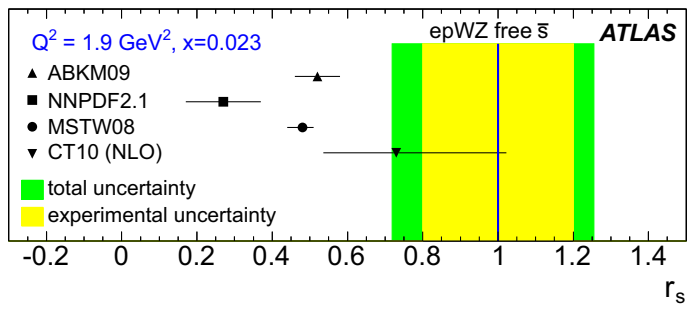

Figure 5. The strange quark suppression factor measured by ATLAS in the $W / Z$ production ratios [9], compared to other determinations.
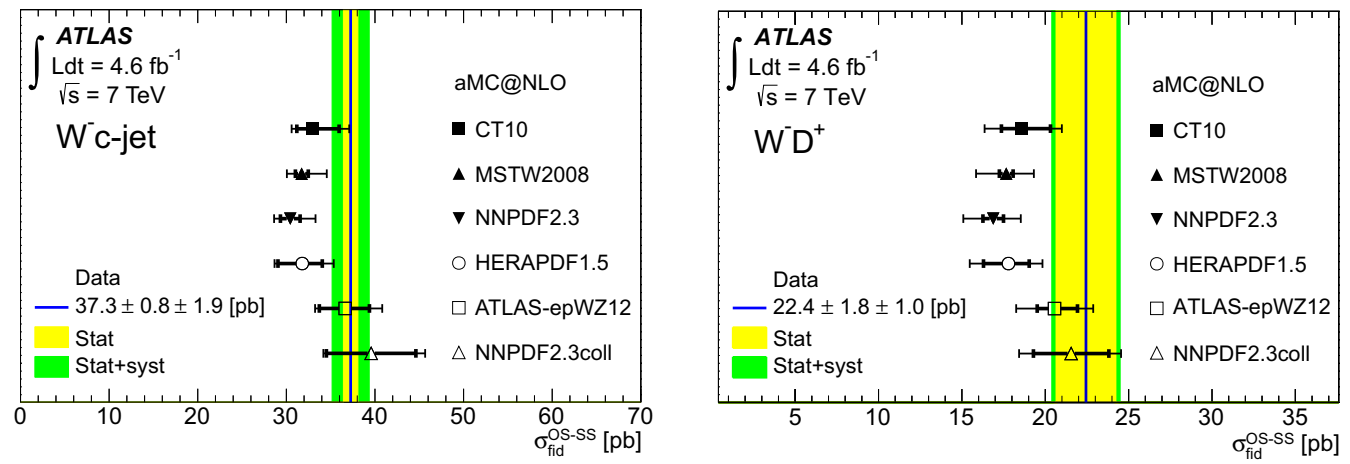

Figure 6. Background-subtracted fiducial cross sections for production of a $W$ boson accompanied by a charm jet and by a $D$ meson [10], compared to predictions.

an example, fig. 7 shows two-dimensional plots with associated uncertainties for $Z$ production and $t \bar{t}$ production. If the theories are calculated at NLO, using corresponding PDF sets, there is substantial disagreement with the data when both channels are considered simultaneously. The theories show some spread, but have large uncertainties. At NNLO, most of the theoretical uncertainties decrease, and the MSTW 2008, CT10, HERAPDF 1.5 (with large uncertainties), NNPDF23 and epWZ models agree well with each other and with the data. The ABM11 and JR09 models now apparently fail.

\section{Conclusions}

The measurements of ATLAS have enlarged the kinematic region over which the proton structure can be studied, and this will improve further when $13 \mathrm{TeV}$ data are available. A variety of models have been presented, and they have varying success in describing the different channels that have been analysed. The precision of the experimental data is an important factor, as is also the precision of the theoretical preductions, which can benefit considerably by extending the model to next-tonext-to-leading order. Different processes have different sensitivities to different PDFs, and the their simultaneous measurement appears to be a powerful technique. At the same time, in any given case, 

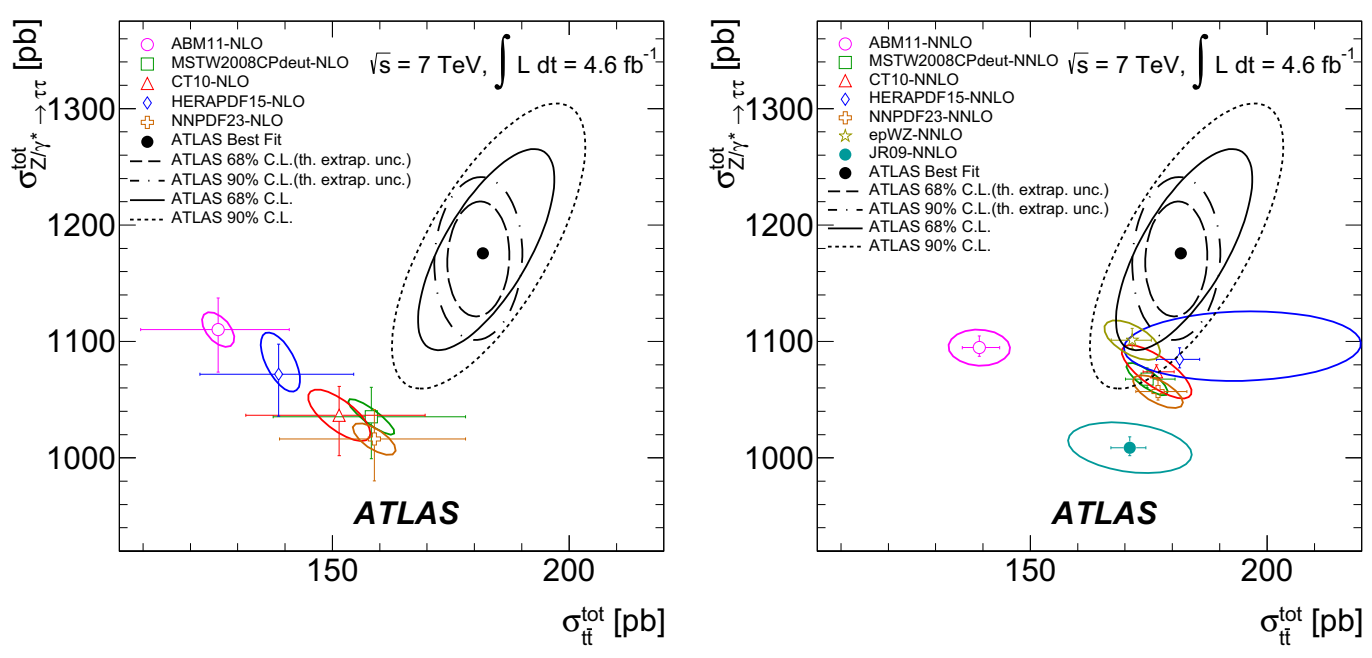

Figure 7. Cross sections for top pair production and $Z$ production plotted simultaneously [11] and compared to theoretical models evaluated at next-to-leading order (left) and next-to-next-to-leading order (right).

it is important to have confidence in the basic dynamical model that is to be used in evaluating the predictions.

With higher energy running with the upgraded ATLAS apparatus, we can expect more precise $b$ tagging, higher statistics in many channels, and improved developments in reconstruction techniques. At the same time, some channels may also have higher backgrounds and tougher running conditions to cope with.

A good knowledge of the proton structure is essential for the accurate measurement of many physical quantities. ATLAS has well demonstrated the importance of this area for present and future work.

\section{References}

[1] ATLAS Collaboration, JINST 3, S08003 (2008)

[2] ATLAS Collaboration, JHEP bf 15, 153 (2015)

[3] ATLAS Collaboration, JHEP bf 14, 059 (2014)

[4] ATLAS Collaboration, Eur. Phys. J. C 75, 228 (2015)

[5] ATLAS Collaboration, Phys. Rev. D 90, 072004 (2014)

[6] ATLAS Collaboration, Phys. Lett. B 725, 223 (2013)

ATLAS Collaboration, JHEP 06, 112 (2014)

[7] ATLAS Collaboration, JHEP 09, 145 (2014)

[8] ATLAS Collaboration, JHEP 10, 141 (2014)

[9] ATLAS Collaboration, Phys. Rev. Lett. 109, 012001 (2012)

[10] ATLAS Collaboration, JHEP 05, 068 (2014)

[11] ATLAS Collaboration, Phys. Rev. D 91, 052005 (2015) 